\title{
Veränderungen der kantonalen Beschäftigtenstrukturen in Industrie und Handwerk in der Schweiz, 1955-1965
}

Hans Elsasser

\section{Einleitung}

Nach Gerfin (1) können die Entwicklungsbesonderheiten in einem Teilraum - im Vergleich zum Gesamtraum - mit Hilfe des Regionalfaktors (R) gemessen werden. Dieser Faktor gibt Auskunft über ein allfälliges Abweichen der Entwicklung in einem Teilgebiet von derjenigen des gesamten Gebietes, d.h. vom Durchschnitt.

Im folgenden sollen nun die Unterschiede im kantonalen Beschäftigtenzuwachs in Industrie und Handwerk während des Zeitraumes von 1955 bis 1965 genauer analysiert werden. Als statistische Unterlagen dienen die Ergebnisse der Eidgenössischen Betriebszählungen von 1955 und 1965 (2).

\section{Statistische Übersicht}

1965 arbeiteten in der Schweiz in Industrie und Handwerk 1142670 Beschäftigte. Das sind 219179 Beschäftigte oder $24 \%$ mehr als zehn Jahre früher (923 491 Beschäftigte).

Die folgenden vier Abbildungen (Abb. 1-4) zeigen - in absoluter und relativer Form - wie sich die Beschäftigtenzahlen in Industrie und Handwerk in den einzelnen Kantonen sowie in den neunzehn Wirtschaftsgruppen 20-38 (Industrie und Handwerk) im Dezenium 1955 bis 1965 verändert haben. Zum Vergleich zwischen den einzelnen Wirtschaftsgruppen müssen zwei Bemerkungen angebracht werden:

1. In den Abbildungen werden als Abkürzungen für die Namen der Wirtschaftsgruppen die offiziellen zweistelligen Nummern 20-38 der Betriebszählung 1965 benützt. Diese bedeuten:

20-38 Industrie und Handwerk

20 Herstellung von Nahrungs- und Futtermitteln

21 Herstellung von Spirituosen und Getränken

22 Tabakindustrie

23 Textilindustrie

24 Herstellung von Kleidern, Wäsche und

Schuhen; Bettwaren

25 Verarbeitung von Holz und Kork (ohne Zimmerei)

26 Kinderwagen, Spielwaren, Sportgeräte

27 Papierindustrie
2. Die Betriebszählung von 1965 wurde grundsätzlich in gleicher Weise durchgeführt wie jene von 1955, und zwar sowohl hinsichtlich der Erhebung selbst wie auch der Aufarbeitung des Zählmaterials. Gegenüber der Zählung von 1955 haben sich folgende die vorliegende Untersuchung betreffenden Änderungen ergeben:

- 1955 existierte noch keine Wirtschaftsgruppe Nr. 32 «Verarbeitung von Mineralöl».

- 1955 gab es eine Wirtschaftsgruppe «Feinmechanik, medizinische Instrumente und Apparate». Diese Wirtschaftsgruppe besaß damals die Nummer 36. 1965 wurde sie in die Wirtschaftsgruppe Nr. 35 «Maschinen, Apparate, Fahrzeuge» integriert.

- Die Wirtschaftsgruppe «Musikinstrumente» umfaßte 1955 auch Musik-, Radio- und Fernsehapparate, welche 1965 teilweise zur Gruppe Nr. 35 "Maschinen, Apparate, Fahrzeuge» gezählt werden. Die Beschäftigtenzahlen von 1955 und 1965 können also in dieser kleinen Wirtschaftsgruppe nicht miteinander verglichen werden.

Die übrigen Abweichungen betreffen in der Regel lediglich die Betriebsarten, d. h. die nächstkleineren Einheiten und nicht die Wirtschaftsgruppen (3).

\section{Der Regionalfaktor}

\subsection{Allgemeines}

Der Regionalfaktor wird folgendermaßen definiert (4): 


$$
\begin{aligned}
& \text { m } 38 \\
& \Sigma \mathrm{b}_{\mathrm{i}}, \mathrm{t} \quad \Sigma \mathrm{b}_{\mathrm{i}, 1965} \\
& \mathrm{i}=1 \\
& \mathrm{~m} \\
& \Sigma b_{i, o} \\
& \mathrm{R}=\frac{\mathrm{i}=1}{\mathrm{~m}} \\
& \Sigma \mathrm{B}_{\mathrm{i}, \mathrm{t}} \\
& \mathrm{i}=1 \\
& \text { m } \\
& \Sigma \mathrm{B}_{\mathrm{i}, \mathrm{o}} \\
& i=1
\end{aligned}
$$

allgemeine Definition

spezielle Definition

\section{$\mathrm{R}=$ Regionalfaktor}

$B_{i}=$ Beschäftigte der Wirtschaftsgruppe $_{\mathrm{i}}$ im Gesamtraum, d. h. im vorliegenden Fall in der Schweiz

$b_{i}=$ Beschäftigte der Wirtschaftsgruppe $_{i}$ im Teilraum, d. h. im vorliegenden Fall in einem Kanton

$0=$ Basisjahr, d. h. im vorliegenden Fall 1955

$\mathrm{t}=$ Endjahr, d. h. im vorliegenden Fall 1965

$\mathrm{m}=$ Anzahl Branchen, d. h. im vorliegenden Fall neunzehn Wirtschaftsgruppen (20-38)
Ist $R=1$, so bedeutet dies, daß sich in einem Kanton die Zahl der Beschäftigten in Industrie und Handwerk zwischen 1955 und 1965 gleich schnell entwickelte wie in der gesamten Schweiz. Ein Wert von $R>1$ heißt, daß in einem Kanton die Beschäftigtenzahl in Industrie und Handwerk, relativ betrachtet, stärker zunahm als in der Schweiz, daß also in diesem Kanton zwischen 1955 und 1965 für die Industrie - gemessen an der Beschäftigtenzahl - wachstumsfördernde Kräfte herrschten. Demgegenüber bedeutet $\mathrm{R}<1$, daß in einem Kanton wachstumshemmende Kräfte wirksam waren.

Eine solche Aussage vermittelte allerdings noch nicht allzuviele Erkenntnisse. Detailliertere Informationen gewinnt man erst, wenn man den Regionalfaktor in einen sogenannten Strukturfaktor und einen Standortfaktor aufspaltet. "Wenn sich nämlich die Industriezweige zwar in der Gesamtwirtschaft verschieden entwickeln, jeder einzelne Industriezweig aber in allen Teilräumen gleich schnell wächst (oder schrumpft), dann beruht die gesamte regionale Entwicklungsbesonderheit allein auf der jeweiligen Branchenstruktur. Standorteinflüsse sind unwirksam. Wenn anderseits in jeder Region die Industriestruktur gleich ist, dann können Entwicklungsunterschiede nur durch Standorteinflüsse ausgelöst sein, die dafür sorgen, daß die einzelnen Zweige nicht überall gleichmäßig expandieren. Hier gibt es keinen Struktureffekt»(5).

Der Regionalfaktor läßt sich somit aufspalten in:

allgemeine Definition:

$$
\begin{aligned}
& \text { m m m } \\
& \sum \mathrm{b}_{\mathrm{i}, \mathrm{t}} \quad \Sigma \mathrm{c}_{\mathrm{i}, \mathrm{t}} \mathrm{B}_{\mathrm{i}, \mathrm{t}} \quad \sum \mathrm{c}_{\mathrm{i},{ }_{\mathrm{o}}} \mathrm{B}_{\mathrm{i}, \mathrm{t}} \\
& \frac{\mathrm{i}=1}{\mathrm{~m}} \quad \frac{\mathrm{i}=1}{\mathrm{~m}} \quad \frac{\mathrm{i}=1}{\mathrm{~m}} \mathrm{~m} \\
& \sum \mathrm{b}_{\mathrm{i}, \mathrm{o}} \quad \sum \mathrm{c}_{\mathrm{i}, \mathrm{o}} \mathrm{B}_{\mathrm{i}, \mathrm{o}} \quad \sum \mathrm{c}_{\mathrm{i}, \mathrm{o}} \mathrm{B}_{\mathrm{i}, \mathrm{o}} \quad \sum \mathrm{c}_{\mathrm{i}, \mathrm{t}} \mathrm{B}_{\mathrm{i}, \mathrm{t}} \\
& \mathrm{R}=\frac{\mathrm{i}=1}{\mathrm{~m}}=\frac{\mathrm{i}=1}{\mathrm{~m}}=\frac{\mathrm{i}=1}{\mathrm{~m}} \times \frac{\mathrm{i}=1}{\mathrm{~m}}
\end{aligned}
$$

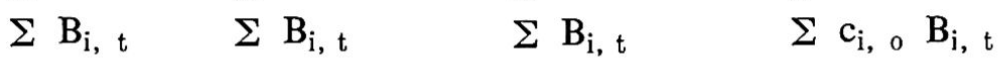

$$
\begin{aligned}
& \begin{array}{llll}
\mathrm{i}=1 & \mathrm{i}=1 & \mathrm{i}=1 & \mathrm{i}=1
\end{array} \\
& \text { m m m } \\
& \Sigma \mathrm{B}_{\mathrm{i}, \mathrm{o}} \quad \Sigma \mathrm{B}_{\mathrm{i}, \mathrm{o}} \quad \Sigma \mathrm{B}_{\mathrm{i}, \mathrm{o}} \\
& \mathrm{i}=1 \quad \mathrm{i}=1 \quad \mathrm{i}=1
\end{aligned}
$$

Regionalfaktor Strukturfaktor Standortfaktor

$c_{i}=$ Anteil des Teilraumes, d. h. im vorliegenden Fall eines Kantons, an den Gesamtbeschäftigten der Branche $_{i}$, d. h. im vorliegenden Fall an einer der neunzehn Wirtschaftsgruppen (20-38) 


\begin{tabular}{|c|c|c|c|c|c|c|}
\hline $\mathrm{ZH}$ & 173901 & $188 \%$ & & $\mathrm{ZH}$ & 212241 & $186 \%$ \\
\hline $\mathrm{BE}$ & 141960 & $154 x_{0}$ & & BE & 168588 & $148 \%$ \\
\hline$A G$ & 82728 & $90 \%$ & & $A G$ & 102728 & $90 \%$ \\
\hline SG & 66904 & $73 \%$ & & SG & 79112 & $69 \%$ \\
\hline so & 53716 & $58 \%$ & & so & 61114 & $54 \%$ \\
\hline vo & 53304 & $58 \%$ & & vo & 72396 & $63 \%$ \\
\hline BS & 46130 & $50 \%$ & & BS & 51811 & $45 \%$ \\
\hline GE & 39039 & $42 \%$ & & GE & 46374 & 41.2. \\
\hline TG & 36277 & $39 \%$ & & TG & 44270 & $39 \%$ \\
\hline NE & 35109 & $38 \%$ & & NE & 41981 & $37 \%$ \\
\hline LU & 34903 & $38 \%$ & & LU & 44186 & $39 \%$ \\
\hline$\pi$ & 26743 & $29 \%$ & & $\pi$ & 40329 & $35 \%$ \\
\hline BL & 26473 & $29 \%$ & & BL & 37390 & $33 \%$ \\
\hline $\begin{array}{l}\text { SH } \\
\text { VS }\end{array}$ & $\frac{16931}{14772} 1 \frac{14758}{1458}$ & $\frac{18 \%}{16 \%}$ & & $\begin{array}{l}\text { SH } \\
\text { VS }\end{array}$ & $\begin{array}{l}21051 \\
22259 \\
2259\end{array}$ & $18 \%$ \\
\hline GL & $\begin{array}{l}\frac{14503}{11093} \\
10912\end{array}$ & 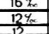 & & $\frac{F R}{-G L}$ & $\begin{array}{l}22024 \\
-11359-\end{array}$ & $\frac{19 q_{r}}{10}$ \\
\hline $3-2=$ & $10268=$ & in= & & $\frac{65}{52}$ & $\begin{array}{l}15151 \\
13755 \\
\end{array}$ & $\frac{14}{12 \% 3}$ \\
\hline $\begin{array}{l}12 G \\
2 \text { ZR } \\
3 \text { UR } \\
4\left\{\begin{array}{l}N W \\
A W\end{array}\right.\end{array}$ & $\begin{array}{l}10061 \\
8246 \\
4208 \\
2174 \\
1997 \\
1304\end{array}$ & 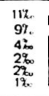 & {$\left[\begin{array}{rl}\text { Beschältigte } \\
\square \\
70000 \\
100 \\
1000\end{array}\right.$} & $\begin{array}{l}Z G \\
A R \\
U R \\
W W \\
N W \\
A I\end{array}$ & $\begin{array}{l}13515 \\
8446 \\
8458 \\
3376 \\
2651 \\
1399\end{array}$ & $\begin{array}{l}122 \times \\
72 \times 0 \\
4 \% \\
3 \% \\
3 \% \\
120\end{array}$ \\
\hline & & 80000 & 50 & & & $1000 \overline{00}$ \\
\hline
\end{tabular}

108

으요.

30\%

408

508

608

708

80\%

908

100:
Betriebszählung
1965

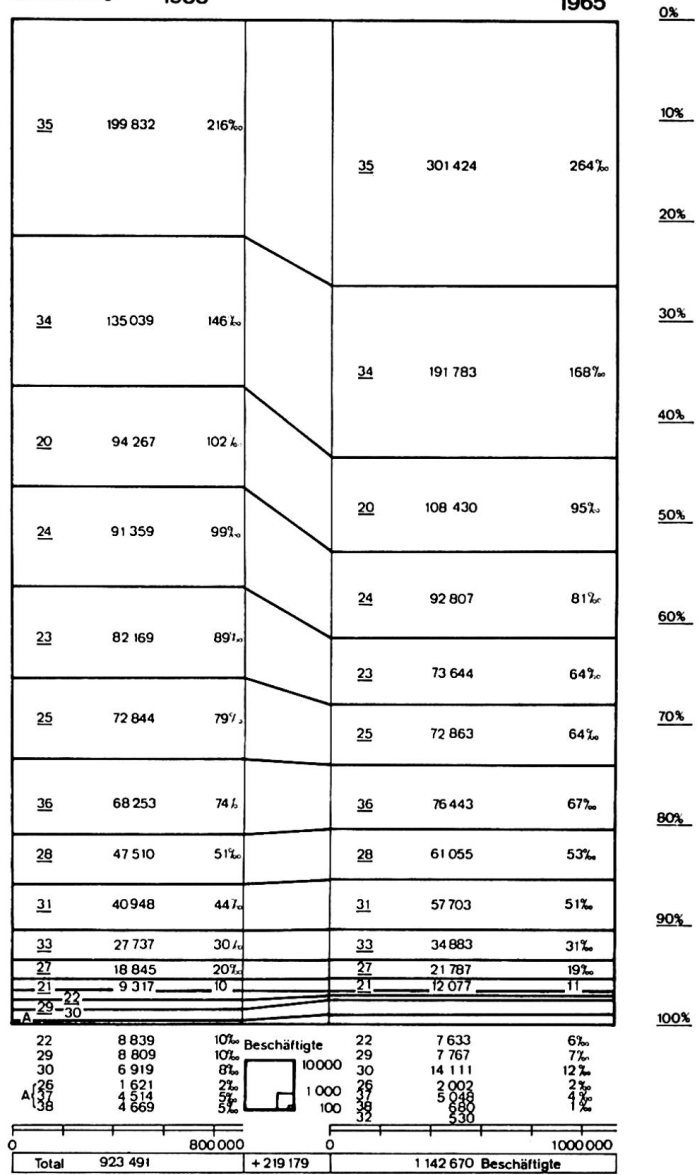

急

108

\%क

so

70\% 


(1)

(2)

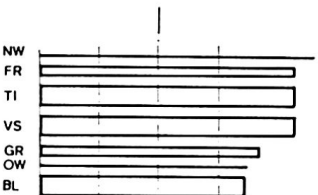

${ }_{\text {vo }}^{\text {Bu }}$

$s_{s 2}^{2 \mathrm{G}}$

(2)

$A G \longdiv { i }$

zH
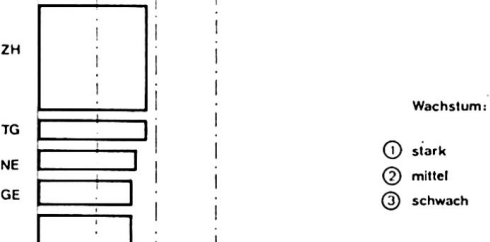

BE

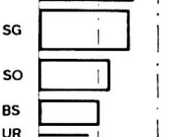

Balkenbreite -
Beschätigle 1965

100000

$\begin{array}{rl}-10000 \\ -5000 \\ E & 2000 \\ 10000 \\ 2000\end{array}$

(3)

un (3) (2) (1)

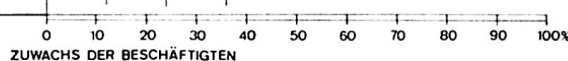

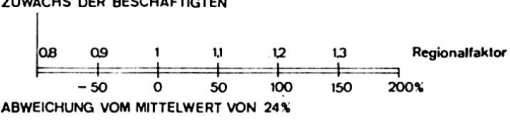

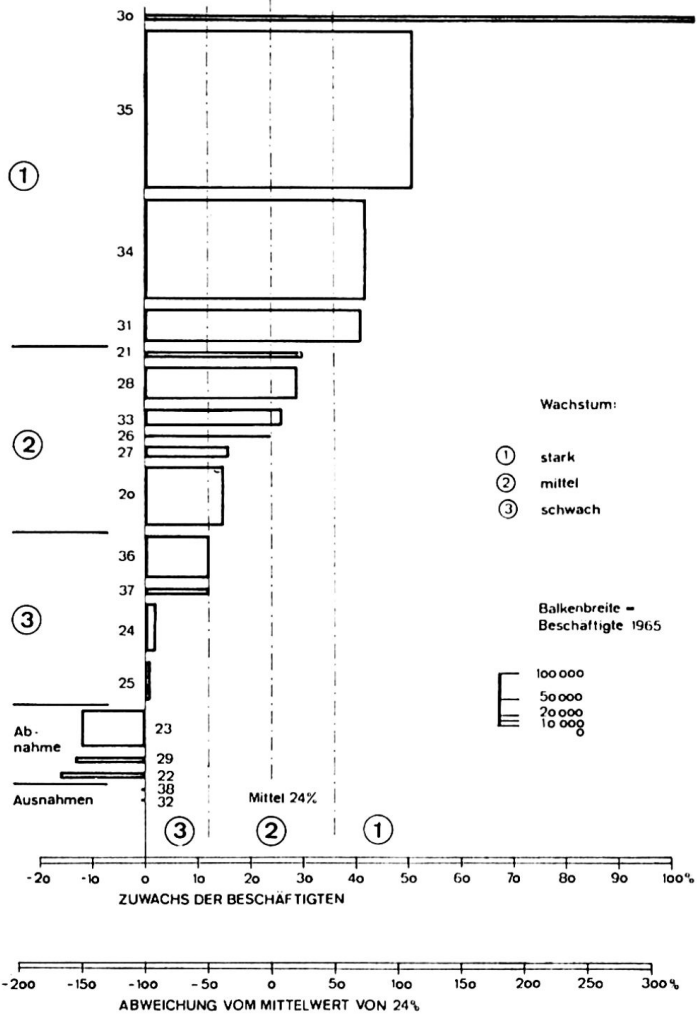


spezielle Definition:

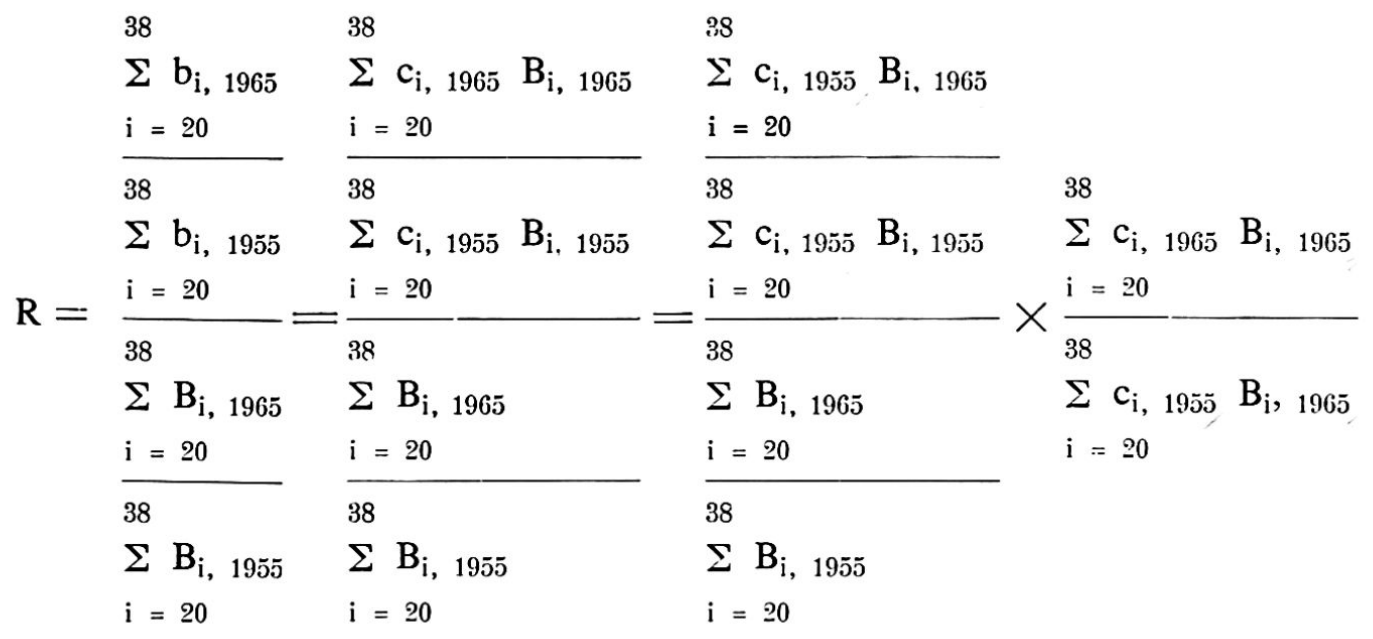

Regionalfaktor Strukturfaktor Standortfaktor

$c_{i}=$ Anteil des Teilraumes, d. h. im vorliegenden Fall eines Kantons, an den Gesamtbeschäftigten de $e_{\perp}$ Branche $_{i}$, d. h. im vorliegenden Fall an einer der neunzehn Wirtschaftsgruppen (20-38)

"Der Standortfaktor wird 1, wenn keine Standorteinflüsse wirksam sind. Überwiegen die relativen Standortvorteile gegenüber den Nachteilen, dann wird der Faktor $>1$ und umgekehrt. Entsprechendes gilt für den Strukturfaktor: Sind die wachstumsintensiven Zweige überdurchschnittlich vertreten, so ist der Faktor $>1$ und umgekehrt» (6).

\subsection{Kritik}

Der Ansatz von Gerfin zur Messung regionalspezifischer Wachstumsunterschiede wurde von verschiedenen Ökonomen, so z. B. von Übe und Schröder (7), diskutiert und kritisiert. Die wichtigsten Einwände gegenüber dem Regionalfaktor und seinen beiden Komponenten sollen hier genannt werden:

- Die Größe des Struktur- und Standortfaktors ist abhängig von der Disaggregation des Untersuchungsmaterials, d. h. im vorliegenden Fall vom Detaillierungsgrad bei der Gliederung des Bereiches von Industrie und Handwerk in neunzehn Wirtschaftsgruppen.

- Eine Aufspaltung des Regionalfaktors kann nur bei größeren Zahlen vorgenommen werden. Úberwiegen in der untersuchten Region ein oder wenige Betriebe, so besteht die Möglichkeit, daß betriebsindividuell bedingte Abweichungen vom Branchendurchschnitt dieses Instrument sinnlos werden lassen. In der vorliegenden Untersuchung ist der Kanton Uri ein solcher Fall.

- Der Begriff "Standortfaktor», ist terminologisch unglücklich gewählt, da dieser Ausdruck auch für die speziellen Standortsvoraussetzungen oder -bedingungen, welche von einem bestimmten Betrieb genutzt werden, verwendet wird (8).

- Der Standortfaktor vermag nicht die Standortgunst eines Teilgebietes, im vorliegenden Fall eines Kantons, im herkömmlichen Sinne darzustellen, sondern er zeigt auf, wie groß die wachstumfördernden oder -hemmenden Einflüsse im Vergleich zum Durchschnitt - auf die regionale Entwicklung sind, die sich nicht durch die branchenmäßige Zusammensetzung der Industrie erklären lassen. All diese Einflüsse werden nun hier als Standortvorteile und -nachteile bezeichnet.

- Der Ansatz von Gerfin eignet sich für ex-postAnalysen, aber im großen und ganzen nicht für Prognosezwecke, da eine Vorausbestimmung des Standortfaktors sehr schwierig ist.

Trotz all dieser Einwände ist es berechtigt und sinnvoll, den Regionalfaktor und seine beiden Komponenten für die fünfundzwanzig Schweizer Kantone aufgrund der Beschäftigtenentwicklung in Industrie und Handwerk zwischen 1955 und 1965 zu berechnen und darzustellen, da eine solche Untersuchung - obwohl die Daten sieben, bzw. siebzehn Jahre alt sind - noch nie veröffentlicht worden ist.

\subsection{Resultate}

Die Berechnungen (9) des Regional-, Struktur- und Standortfaktors haben für die Wirtschaftsgruppen 
des Bereiches Industrie und Handwerk von 1955 bis $1965 \mathrm{zu}$ folgenden kantonalen Resultaten geführt:

\begin{tabular}{|c|c|c|c|}
\hline 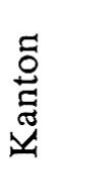 & 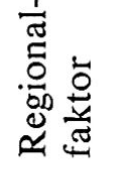 & 莺 & 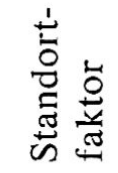 \\
\hline $\mathrm{ZH}$ & 0,987 & 1,033 & 0,955 \\
\hline $\mathrm{BE}$ & 0,960 & 0,992 & 0,968 \\
\hline LU & 1,024 & 0,988 & 1,036 \\
\hline UR & 0,896 & 1,155 & 0,779 \\
\hline SZ & 1,083 & 0,926 & 1,170 \\
\hline OW & 1,147 & 0,886 & 1,295 \\
\hline NW & 1,255 & 0,978 & 1,283 \\
\hline GL & 0,828 & 0,897 & 0,923 \\
\hline ZG & 1,086 & 1,065 & 1,020 \\
\hline FR & 1,222 & 0,981 & 1,246 \\
\hline SO & 0,920 & 1,010 & 0,911 \\
\hline BS & 0,908 & 1,052 & 0,863 \\
\hline BL & 1,142 & 1,092 & 1,107 \\
\hline $\mathrm{SH}$ & 1,005 & 1,058 & 0,950 \\
\hline AR & 0,825 & 0,908 & 0,909 \\
\hline AI & 0,867 & 0,839 & 1,034 \\
\hline SG & 0,956 & 0,920 & 1,039 \\
\hline GR & 1,166 & 0,964 & 1,210 \\
\hline$A G$ & 1,003 & 1,006 & 0,997 \\
\hline TG & 0,986 & 0,960 & 1,027 \\
\hline TI & 1,220 & 0,943 & 1,294 \\
\hline VD & 1,097 & 0,995 & 1,103 \\
\hline VS & 1,217 & 1,044 & 1,166 \\
\hline NE & 0,966 & 0,975 & 0,991 \\
\hline $\mathrm{GE}$ & 0,960 & 1,038 & 0,925 \\
\hline$(\mathrm{CH}$ & 1,000 & 1,000 & $1,000)$ \\
\hline
\end{tabular}

Diese Resultate können nicht direkt mit den von Doerig (10) berechneten Werten verglichen werden, da sich dieser Autor einerseits auf die Daten der Volkszählungen 1941, 1950 und 1960 stützte und anderseits seine Faktoren mit den Werten von fünfzehn verschiedenen Branchen des Sekundärsektors (einschl. Bergbau, Baugewerbe sowie Elektrizität, Gas und Wasser) errechnete.

\section{Interpretationen}

Die Berechnungen und kartographischen Darstellungen (Abb. 5-8) des Regional-, Struktur- und Standortfaktors vermitteln ein aufschlußreiches Bild über die Entwicklung der Beschäftigten in Industrie und Handwerk in allen schweizerischen Kantonen. In den folgenden Abschnitten soll nun versucht werden, diese regional unterschiedlichen Größen zu erklären.

\subsection{Strukturfaktor}

Dieser Faktor gibt an, in welchen Kantonen besonders wachstumstarke Industrien - gemessen an der Beschäftigtenzahl - beheimatet und wo eher schwache Branchen vertreten sind. "Im Durchschnitt erklärt der Strukturfaktor rund die Hälfte der gesamten regionalen Abweichung, wenn nicht allzu kleine Teilräume betrachtet werden» (11).

Die Spanne der Kantone reicht von 1,155 des Kantons Uri bis zu 0,839 von Appenzell-Innerrhoden. Der wohl etwas überraschend hohe Wert von Uri läßt sich darauf zurückführen, daß in diesem Kanton die drei wachstumintensivsten Wirtschaftsgruppen "Kunststoffverarbeitung», "Maschinen-» und "Metallindustrie» 67\% aller in Industrie und Handwerk Beschäftigten umfassen, währenddem der entsprechende Wert für die gesamte Schweiz lediglich $43 \%$ beträgt. Ähnliche Verhältnisse finden wir auch im Kanton Wallis. In diesem Kanton arbeiten $63 \%$ der Beschäftigten von Industrie und Handwerk in der Metall-, Maschinen- und der chemischen Industrie, im Gegensatz zur übrigen Schweiz, wo es $48 \%$ sind. Die anderen Regionen, in denen wachstumstarke Industriezweige überdurchschnittlich vorkommen, sind einerseits der Kanton Genf und anderseits das große Gebiet der Nordwest- und Nordostschweiz.

Die Mehrzahl der Kantone, nämlich fünfzehn, besitzen einen unterdurchschnittlichen Strukturfaktor. Die tiefsten Werte finden sich in Kantonen mit einem hohen Anteil an Beschäftigten in der Textilindustrie (Ostschweiz), in der holzverarbeitenden Industrie (Obwalden, Schwyz und Graubünden) sowie in der Bekleidungsindustrie (Tessin). Auch die beiden "Uhrenkantone» Neuenburg und Bern, in welchen zusammen 1965 62\% aller Beschäftigten der schweizerischen Uhrenindustrie arbeiteten, gehören zu den Kantonen mit einem Strukturfaktor $<1$.

\subsection{Standortfaktor}

Der Standortfaktor zeigt auf, in welchen Kantonen die branchenstrukturbereinigten Standortvorteile überwiegen und umgekehrt. $\mathrm{Zu}$ diesen Vor- und Nachteilen zählen - wie bereits erwähnt - nicht nur die bekannten herkömmlichen Standortvoraussetzungen: «Natürliche Voraussetzungen», «Verkehrsmäßige Erschließung», "Agglomerations- und Fühlungsvorteile» sowie "Arbeitsmarktsituation» (12), sondern ebensosehr kantonale Maßnahmen zur Förderung der Industrieansiedlung wie auch besondere Unternehmerfähigkeiten in den einzelnen Regionen u.ä.

Vierzehn Kantone besitzen einen Standortfaktor $>1$. Diese Kantone liegen in der Ost- und Zen- 

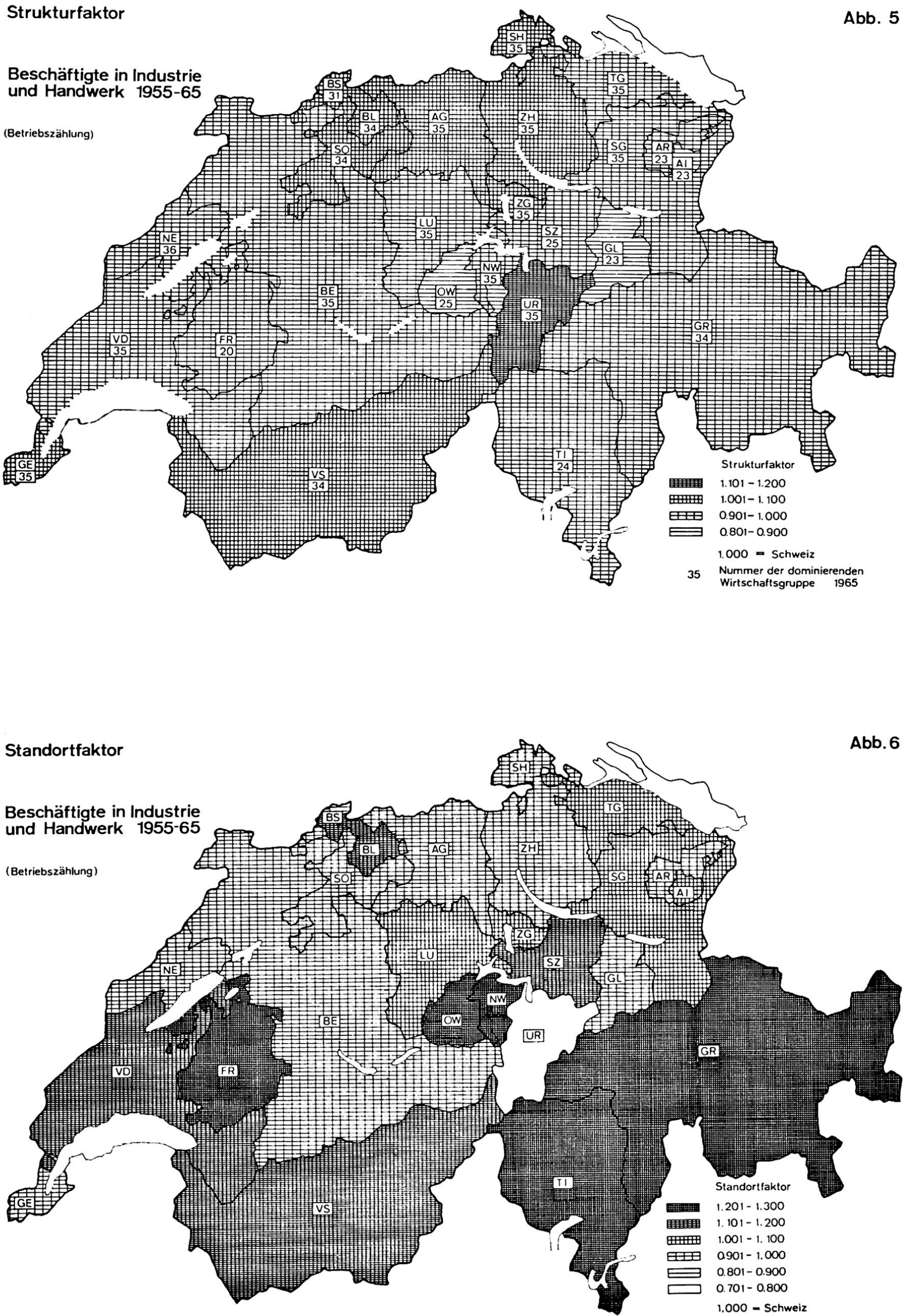


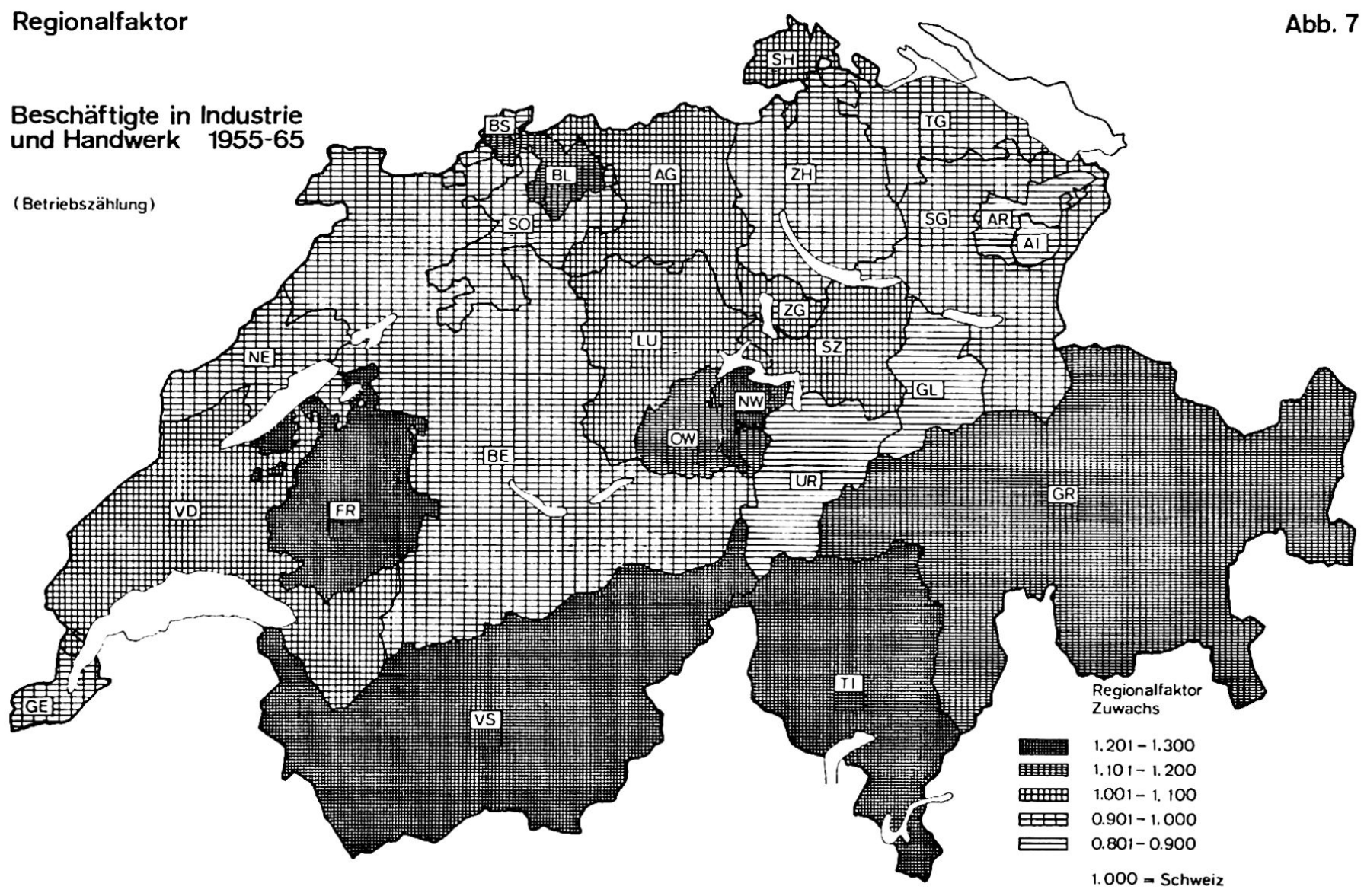

Regional-, Standort- und Strukturfaktor

Abb. 8

Beschäftigte in Industrie und Handwerk 1955-65 (Betriebszählung)

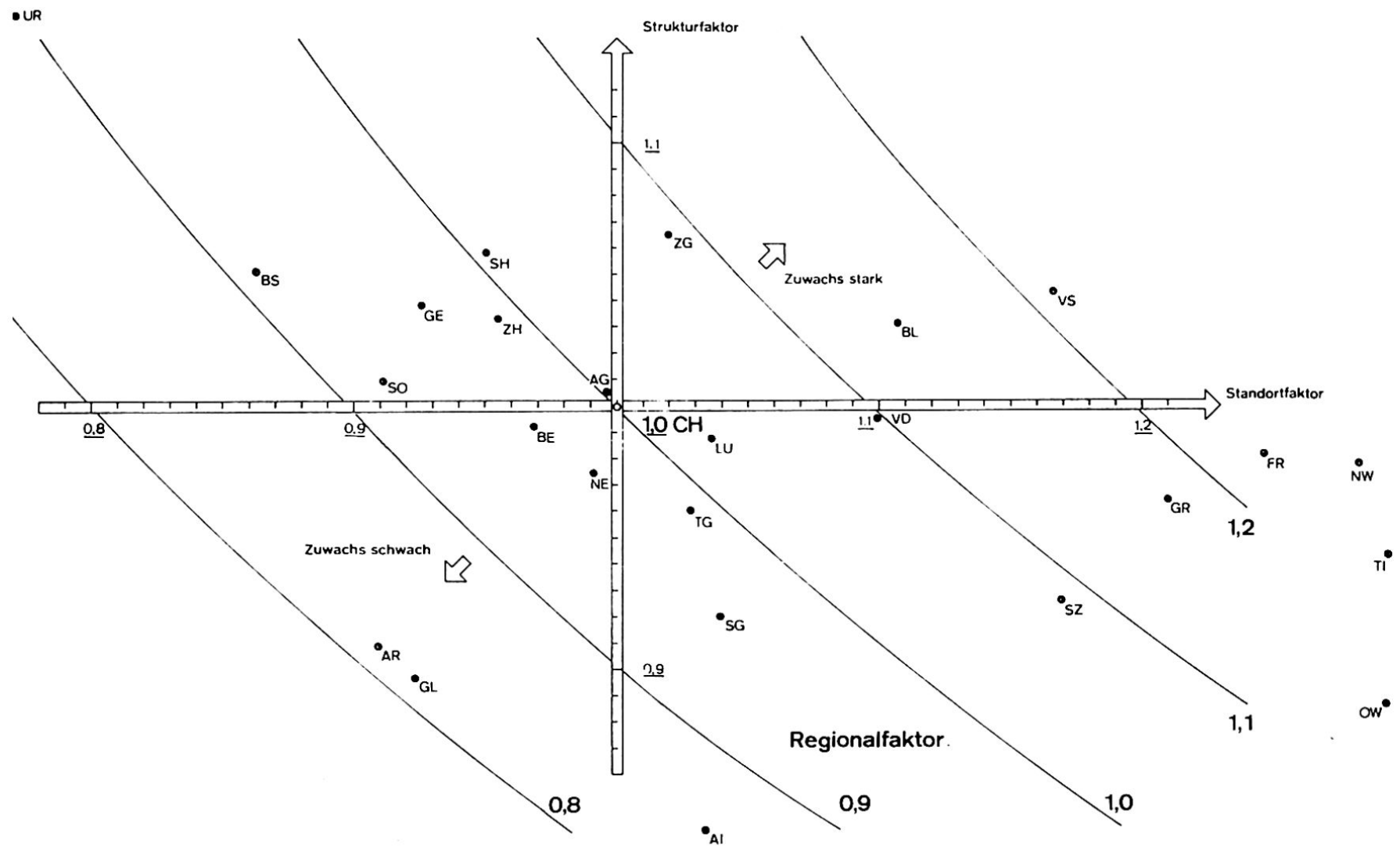


tralschweiz sowie im alpinen Raum (Ausnahmen: Uri, Glarus und Appenzell-Außerrhoden). Dazu kommen noch die beiden welschen Kantone Freiburg und Waadt. Die unterschiedlichen Werte von Basel-Stadt und Baselland lassen sich darauf zurückführen, daß die wachstumintensive Industriestruktur in der Stadt Basel (Strukturfaktor 1,052) zu räumlichen Sättigungserscheinungen führte. Die darauf einsetzende Verlagerung in das Umland, d.h. in den Kanton Baselland, drückt sich in einem niedrigen Standortfaktor für das Kerngebiet und einem hohen für das Randgebiet aus (13). Überdurchschnittliche Standortfaktoren weisen im Prinzip alle jene Kantone auf, in denen ein «industrieller Nachholbedarf» besteht, d.h. die meisten alpinen und voralpinen Kantone, währenddem sich in den übrigen Kantonen mit einem hohen Anteil von Beschäftigten in Industrie und Handwerk z. B. Zürich, Solothurn, Schaffhausen und Aargau gewisse Sättigungserscheinungen auswirken. Es handelt sich hier in erster Linie um Sättigungserscheinungen bezüglich der Arbeitskraftreserven. Zwischen dem Industrialisierungsgrad, ausgedrückt durch die Zahl der Beschäftigten in Industrie und Handwerk pro Einwohner (14), und dem Standortfaktor besteht folgende Abhängigkeit (15): Ein relativ geringer (großer) Industrialisierungsgrad führt zu einem relativ großen (geringen) Standortfaktor: negative Korrelation. Die Chance für relative Standortverbesserungen ist in Räumen mit geringem Industrialisierungsgrad größer als in industriellen Ballungsgebieten (16).

Die relativ hohen Standortfaktoren in den Kantonen Obwalden, Freiburg und Wallis beispielsweise lassen sich aber auch auf Erfolge von kantonalen und kommunalen Maßnahmen zur Förderung der Industrieansiedlung in diesen Gebieten zurückführen. Der Standortfaktor des Tessins ist eine Folge des starken Zuzuges ausländischer Arbeitskräfte, d.h. vor allem Grenzgänger im Mendrisiotto.

\subsection{Regionalfaktor}

Die Multiplikation von Struktur- und Standortfaktor ergibt den Regionalfaktor, der aufzeigt, in welchen Kantonen die Zahl der Beschäftigten in Industrie und Handwerk über- oder unterdurchschnittlich zugenommen hat.

$\mathrm{Zu}$ den dreizehn Kantonen mit einem überdurchschnittlichen Beschäftigtenwachstum gehören Gebirgskantone oder Kantone mit größeren alpinen Gebieten, wie Nidwalden, Freiburg, Wallis, Graubünden, Obwalden und Schwyz. Nicht dazu zählen die bedeutenden Industriekantone Zürich, Bern, St. Gallen und Solothurn. Dies zeigt deutlich, daß in der Zeitspanne von 1955 bis 1965 nicht von einer weiteren räumlichen Konzentration der Beschäftigten in Industrie und Handwerk gesprochen werden kann, sondern daß sich Dezentralisationserscheinungen abzeichneten, sofern die Kantone als kleinste statistische Einheiten für die Unterteilung der Schweiz gewählt werden. Die Ursachen dieser Dezentralisierungstendenzen können mit den Begriffen «industrieller Nachholbedarf» und «industrielle Sättigung» knapp charakterisiert werden.

Die Kombination von Struktur- und Standortfaktor ergibt vier Möglichkeiten:

1. Die industriewirtschaftliche Situation - gemessen an der Beschäftigtenentwicklung - muß in den Kantonen Zug, Baselland und Wallis als sehr gut bezeichnet werden, besitzen doch diese Kantone sowohl einen überdurchschnittlichen Struktur- als auch einen ebensolchen Standortfaktor.

2. Im Gegensatz dazu kennzeichnen sich die beiden "Uhrenkantone» Neuenburg und Bern sowie die beiden "Textilkantone» Appenzell-Außerrhoden und Glarus nicht nur durch einen überdurchschnittlichen Anteil an beschäftigungsmäßig bloß bescheiden expandierenden, stagnierenden oder gar schrumpfenden Branchen aus, sondern jene Wirtschaftszweige, die sich im gesamtschweizerischen Raum als wachstumstarke Industrie- und Gewerbegruppen erweisen, entwickeln in diesen Kantonen nicht ihre volle Kraft. Diese Situation führte - neben anderen wirtschaftlichen Entwicklungen - im Kanton Bern dazu, daß ein Gesetz über die Förderung der Wirtschaft erlassen wurde, welches vom Souverän am 12. Dezember 1971 angenommen wurde.

3. Die Industriekantone Zürich, Solothurn, BaselStadt, Schaffhausen, Aargau und Genf besitzen alle eine gute industrielle Branchenstruktur, aber es wirken sich hier Sättigungserscheinungen aus, welche dazu führen, daß - mit Ausnahme von Schaffhausen und des Aargaus - nicht nur der Standort- sondern auch der Regionalfaktor $<1$ wird. Zu dieser Gruppe zählt auch der Kanton Uri. Hier kann allerdings der sehr niedrige Standortfaktor $(0,779)$ nicht auf industrielle Sättigung zurückgeführt werden, sondern viel eher auf die relativ ungünstigen Standortsvoraussetzungen für die Industrie. Im Gegensatz zu anderen Gebirgskantonen, z. B. Wallis, wurden in Uri zwischen 1955 und 1965 keine großen, gezielten, von Erfolg gekrönten Anstrengungen unternommen, um neue Industriebetriebe für eine Ansiedlung zu gewinnen. Ferner muß berücksichtigt werden, $\mathrm{da} \beta$ in diesem Kanton 1965 über die Hälfte aller Beschäftigten in Industrie und Handwerk in zwei Unternehmungen (drei Betrieben) arbeiten, daß 
sich also hier unternehmungs- und betriebsspezifische Besonderheiten sehr stark auswirken.

4. Die meisten Kantone befinden sich in der Gruppe «Niedriger Strukturfaktor - Hoher Standortfaktor». Diese Kombination zeigt, daß in diesen Kantonen nicht Wachstumsindustrien angezogen wurden, sondern vielmehr arbeitsintensive, zum Teil sogar stagnationsbedrohte Zweige, die durch Verlagerung versuchen, dem permanenten Kostendruck in den übrigen Regionen zu entweichen. Das bedeutet natürlich keinesfalls, daß jeder sich in diesen Kantonen neu ansiedelnde Betrieb zu dieser Kategorie gehört.

Solche Kantone zeigen häufig zunächst einen wirtschaftlichen Aufschwung, d. h. ihr Regionalfaktor ist $>1$, aber sie vergrößern nur ihren Anteil an schwachen Industriezweigen. Ihre Standortkomponente ist vorübergehend günstig, aber ihre Industriestruktur verschlechtert sich zunehmend. Das beweist mit allem Nachdruck, $\mathrm{da} ß$ eine längerfristige Wirtschaftsförderung nicht nur durch Verbesserung der Standortbedingungen erfolgen darf, sondern da $\beta$ auch die branchenmäßige Zusammensetzung der Industrie sorgfältig beachtet werden muß. "Man sollte nicht einfach jede erreichbare Ansiedlung akzeptieren, sondern eine genaue Analyse vornehmen, was der Region langfristig nützen kann» (17). Sonst besteht die Gefahr, daß nach einiger Zeit die Erfolge der Wirtschaftsförderung wieder dahin sind.

\section{Zusammenfassung}

Im Zeitraum 1955 bis 1965 hat die Zahl der Beschäftigten in Industrie und Handwerk vor allem in sehr schwach industrialisierten Kantonen überdurchschnittlich zugenommen, währenddem die traditionellen Industriekantone zum großen Teil ein eher unterdurchschnittliches Wachstum verzeichneten. Es kann deshalb für dieses Dezennium nicht von einer weitergehenden räumlichen Konzentration der Beschäftigten in Industrie und Handwerk gesprochen werden. Diese Wachstumsunterschiede lassen sich auf die regional unterschiedliche Branchenstruktur der Industrie und die verschiedenen industriellen Standortvoraussetzungen im weitesten Sinne zurückführen. Bei der Förderung der Industrieansiedlung durch die öffentliche Hand muß beachtet werden, daß nicht nur die industriellen Standortvoraussetzungen verbessert werden, sondern auch die branchenmäßige Zusammensetzung der Industrie spielt eine entscheidende Rolle, um bleibende Erfolge der Wirtschaftsförderung zu erzielen.
1 Gerfin, H.: Gesamtwirtschaftliches Wachstum und regionale Entwicklung. In: Kylos, Vol. XVII (1964), S. 565-592.

2 Vgl. dazu Elsasser, H.: Bemerkungen zu einigen industriewirtschaftlichen Begriffen. In: DISP, Nr. 22 (1971), S. 18-21.

3 Für weitere Details siehe Band 3 «Betriebe, Hauptergebnisse nach Kantonen» der Eidgenössischen Betriebszählung, September 1965, Industrie und Gewerbe. Eidgenössisches Statistisches Amt, Bern 1967, Statistische Quellenwerke der Schweiz/Heft 411, Reihe Df 3, S. 711.

4 Nach Gerfin, H.: a. a. O., S. 582.

5 Gerfin, H.: a. a. O., S. 585.

6 Gerfin, H.: a. a. O., S. 586.

7 Ube, W.: Industriestruktur und Standort. Prognos Studie 1 (1967), Stuttgart, S. $53 \mathrm{ff}$.

Schröder, D.: Strukturwandel, Standortwahl und regionales Wachstum. Prognos Studie 3 (1968), Stuttgart, S. $61 \mathrm{ff}$.

8 Rüschenpöhler, H.: Der Standort industrieller Unternehmungen als betriebswirtschaftliches Problem. Abhandlungen aus dem Industrieseminar der Universität zu Köln, Heft 6 (1958), Berlin, S. 64 ff.

9 Berechnungen durchgeführt von R. Abt, lic. oec. publ., ORL-Institut ETHZ.

10 Doerig, H. U.: Der staatliche Einfluß auf die regionale Verteilung von Bevölkerung und Wirtschaft. Diss. HSG. Zürich und St. Gallen 1968, Tab. 7, S. 41.

11 Gerfin, H.: a. a. O., S. 587.

12 Vgl. dazu Elsasser, H.: Die Standortvoraussetzungen für die Industrie in der Schweiz. In: Wi. pol. Mit. 1, 1971.

13 Vgl. dazu Gerfin, H.: a. a. O., S. 589.

14 Vgl. dazu Elsasser, H. und Rouge, C.: Industriebesatz 1970. ORL-Studienunterlage Nr. 10 (1972), ORL-Institut ETHZ, S. 3.

$15 \mathrm{r}=-0.513$ (Spearmanscher Rang-Korrelationskoeffizient), berechnet für die fünfundzwanzig Kantone mit den Werten "Standortfaktor» und "Industrialisierungsgrad 1965».

16 Vgl. dazu Übe, W.: a. a. O., S. 67.

17 Gerfin, H.: a. a. O., S. 590.

Adresse des Verfassers:

PD Dr. Hans Elsasser

Institut für Orts-, Regional- und Landesplanung

der ETH

Weinbergstraße 35, 8006 Zürich 